\title{
Il castello di Tutino (Le): una lettura storico-architettonica per la conoscenza del patrimonio pugliese
}

The castle of Tutino (Le): a case study on knowledge of the Apulian heritage

\author{
Alessandra Ponzetta \\ Sapienza Università di Roma, Rome, Italy, ponzetta.alessandra@gmail.com
}

\begin{abstract}
This study aims to investigate the relationship between a castle located in the Apulian region (Southeastern Italy) and its historical and territorial background. The subject of the research deals with a multilayered monumental complex located in the lower Salento, in the territory of the town of Tricase, which includes five castles. This currently presents itself as an irregularly shaped fence marked by five towers, whose original structure dates back to at least the fifteenth century. In particular, on one side of the castle a baronial palace was built by the Trani family in the eighties of the sixteenth century. As far as it concerns the history of the entire complex it should be noticed that it has undergone various enlargements and modifications due to changes of its property and use. This process is both documented at the archival-bibliographic level, and experienced by the analysis of the masonry stratigraphy, thanks to which it has been possible to identify the various stages of the historical-constructive development of the castle. In conclusion, the analysis carried out intends to clarify how the historical dynamics occurred in the region of Apulia influenced the final stage of the castle of Tutino; to this end it will be considered the evolution of constructive techniques and perspectives belonging to the local tradition, in order to demonstrate the impact on the features of this castle.
\end{abstract}

Keywords: Castle of Tutino, Trani palace, Apulian heritage, architectural restoration.

\section{Introduzione}

Il contributo che qui si propone intende gettare nuova luce su uno dei molti esempi minori di architettura fortificata presente nel territorio pugliese. In Puglia, infatti, quasi ogni centro urbano risulta caratterizzato dalla presenza di un castello o di una torre, non soltanto le realtà più grandi ed importanti, ma anche quelle più piccole e meno conosciute. Attraverso la ricerca bibliografico-archivistica e l'indagine diretta sul bene, l'intento di questo lavoro è pertanto quello di implementare la conoscenza di questo patrimonio al fine di incentivare attività di conservazione e valorizzazione più consapevoli. Oggetto di questo approfondimento ${ }^{1}$ è il castello di Tutino (o palazzo dei Trani), un complesso architettonico pluristratificato situato nell'estremo Salento, nel territorio di Tricase. Esso, infatti, non costituisce soltanto un esempio di architettura fortificata in Terra d'Otranto, in particolar modo della tipologia a recinto, ma rappresenta al tempo stesso una testimonianza di quel processo, verificatosi in Puglia come altrove tra Cinquecento e Seicento, di trasformazione di vecchi manieri in residenze gentilizie, una volta persa l'originaria funzione di difesa e di controllo. 


\section{Note sul contesto storico-territoriale}

L'analisi del contesto storico-territoriale di appartenenza fornisce le prime informazioni utili ad una maggiore conoscenza del caso studio. Il castello di Tutino è infatti situato nel piccolo rione, da cui prende il nome, posto a nord-ovest di Tricase. Questo centro risulta costituito dall'unione di più nuclei urbani, ognuno dei quali è caratterizzato dalla presenza di un castello, da cui l'appellativo di "Terra dei cinque castelli”. Si possono contare: 1) il castello o palazzo dei Principi Gallone nel centro storico di Tricase; 2) il castello o palazzo baronale dei Trani nel rione di Tutino (a nord-ovest); 3) il castello dei conti del Balzo nel rione di Caprarica del Capo (a sud); 4) il castello o palazzo baronale dei Winspeare nella frazione di Depressa (a nord); 5) il castello o palazzo baronale degli AlfaranoCapece nella frazione di Lucugnano (a ovest).

Interessante è soffermarsi sul motivo che ha portato alla realizzazione di così tante fortificazioni, abbastanza vicine tra loro in un territorio così poco esteso. Sicuramente esso è da ricercare in primo luogo nella connotazione idrogeomorfologica del territorio in rapporto agli eventi storici e alle dinamiche di insediamento che hanno caratterizzato la regione. Se si considera l'orografia del sito, questo territorio appare caratterizzato dalla presenza delle cosiddette serre salentine, elementi collinari caratterizzati da allineamenti di modeste groppe sassose, tra cui si creano piccole valli, zone depresse e pianeggianti ricche di acqua. La lettura dell'idrografia del sito (Arditi, 1979, p. 631), pertanto, aiuta a comprendere come in passato la presenza di pozzi in queste zone abbia favorito l'insediamento umano, con l'addensarsi di numerosi centri abitati di piccole dimensioni in aree molto vicine tra loro. Inoltre, l'ubicazione di questi centri in prossimità della costa, elemento che li rendeva facile bersaglio delle numerose incursioni a cui questo territorio era soggetto, ha sicuramente favorito la nascita di architetture fortificate, utili e necessarie in caso di assedio. L'attenta lettura del territorio ha inoltre consentito l'individuazione di una serie di percorsi storici lì dove l'urbanizzazione moderna non ne ha ancora cancellato le tracce. In particolare, è possibile riscontrare due tracciati nelle traiettorie principali di attraversamento di questo territorio: un percorso trasversale (est-ovest), che dalla costa orientale metteva probabilmente in collegamento Tricase, Tutino, Lucugnano, e attraverso il territorio di Specchia, posizionata su un'altura e dotata anch'essa di un castello, arrivava fino ad Ugento ad occidente; un altro tracciato longitudinale (nord-sud), che passando per Tricase e per Caprarica, collegava Castro, a settentrione, con il Capo di Leuca all'estrema propaggine meridionale.

Nonostante i cinque castelli di Tricase non facciano parte di un progetto di fortificazione unitario e non esistano documenti che ne attestino un esplicito legame reciproco, è tuttavia possibile condurre una serie di osservazioni sulle loro relazioni, anche in merito alla specifica tipologia costruttiva. Va infatti osservato come sia il castello di Tutino, sia quello di Caprarica del $\mathrm{Ca}$ $\mathrm{po}^{2}$, costituiscano due esempi di recinto fortificato (Cazzato, Cazzato, 2005, p. 87) e risultino posizionati quasi alla stessa distanza rispetto al centro di Tricase. Diversamente, i castelli di Lucugnano e Depressa, assieme a quello di Tricase, nascono da un nucleo primitivo costituito da una torre e si localizzano ad una distanza maggiore, ma sempre equidistante rispetto al centro di Tricase. Tali osservazioni permettono di valutare come alla differente tipologia costruttiva corrisponda un diverso ruolo strategico nel territorio: infatti, se i recinti di Tutino e di Caprarica del Capo fungevano da rifugio per le popolazioni in caso di assedio; Tricase, Lucugnano e Depressa, sorti e sviluppatisi intorno a torri preesistenti, avevano una funzione prettamente difensiva, di avvistamento e di controllo su un territorio più vasto. Questo spiegherebbe anche la loro collocazione a distanze fisse, ma differenti, rispetto al centro maggiore di Tricase nell'ottica di una diversa funzione sul territorio.

\section{Le origini del castello di Tutino}

Nonostante certa letteratura attribuisca il castello di Tutino al periodo svevo (Fuzio, 1979, p. 153), il primo documento che fa esplicito riferimento ad esso risale al $1444^{3}$. Ciò consente di affermare ragionevolmente che a quella data il castello esistesse già; tuttavia, l'assenza di ulteriori dati 
non permette di riferirne la costruzione ad uno specifico periodo storico. Va detto, comunque, che nessuna delle fonti visionate relativamente agli anni precedenti al 1444 menziona Tutino come castello o castrum, ma sempre come casa$l e$. Tale osservazione lascia pensare che il castello possa essere stato costruito proprio negli anni di sottoscrizione del documento, sotto la famiglia del Balzo. Questa, infatti, rappresentava una delle più prestigiose famiglie nobiliari del meridione, per lungo tempo al governo del principato di Taranto ed impegnata in diverse opere di fortificazione sul territorio ${ }^{4}$.

Se già appare difficile individuare un periodo di fondazione preciso per questo castello, ancora più difficoltoso risulta immaginare quali fossero le sue fattezze originarie (Fig. 1). A tal proposito Cosimo De Giorgi (Giorgi, 1882-1888, p. 166) riferisce che "nel XVI sec. nove torri quadre coronate di merli difendevano la terra di Tutino", mentre nell'epoca in cui scriveva ne restavano solo cinque. Mario Cazzato (Cazzato, 1997, p. 142) sostiene, invece, che in origine il castello dovesse avere un impianto a recinto quadrangolare con tre torri per lato, di cui due angolari, munite probabilmente nella seconda metà del XV secolo di un'estremità inferiore scarpata e di un coronamento a mensole a sorreggere un modesto sporto.

Gli studi condotti consentono di essere piuttosto certi nel dire che dovesse trattarsi di un recinto fortificato - una tipologia molto diffusa nel territorio salentino- costituito da una cortina muraria a delimitazione di uno spazio interno, lungo la quale si innestano torri dalla forma quadrangolare o circolare. L'analisi effettuata con il georadar in occasione di questo lavoro, sul versante sudoccidentale nel punto in cui le mura si allargano verso ovest, fa tuttavia escludere l'ipotesi di un originario recinto quadrangolare di impianto regolare; non ci sono infatti tracce di un tratto murario che prosegua rettilineo verso il lato nordoccidentale del castello. Il castello, dunque, deve aver sempre avuto questa configurazione irregolare, dettata probabilmente dalla presenza di un banco di roccia sottostante con tale andamento, sul quale si è posizionato.
Per quanto riguarda, invece, le torri attualmente presenti, quanto riscontrato attraverso l'analisi stratigrafica induce a considerare: le prime due lungo il versante nord-orientale, di dimensioni minori e non scarpate, come le più antiche; mentre le ultime due sul lato sud-orientale, di dimensioni maggiori, scarpate e coronate da mensole, come le più recenti. Queste ultime, infatti, assieme alla torre posta in angolo tra $\mathrm{i}$ due fronti, la quale rappresenta un ibrido poiché simile alle più antiche nelle dimensioni (larghezza e altezza), ma con l'estremità inferiore scarpata come le più recenti, devono aver subito un rifacimento nel corso del tempo. Questo dev'essere consistito nel rivestimento dell'originaria struttura con una nuova, che presentava una base scarpata e, nel caso delle ultime due torri sul fronte sudorientale, anche un coronamento a mensole. Sulla base di quanto sostenuto anche da Mario Cazzato (Cazzato, 1997, p. 142), si può ipotizzare che tale intervento possa essere avvenuto in concomitanza con un periodo di maggiore allerta dovuto alle invasioni dei Turchi, che nel 1480 conquistano Otranto. ${ }^{5}$

L'indagine stratigrafica è stata fondamentale anche per ipotizzare l'esistenza di un cammino di ronda all'interno del castello. L'osservazione diretta e attenta del manufatto, infatti, ha consentito di identificare nelle torri dei conci di ammorsamento che dovevano collegarle in origine con la cinta muraria, di cui alcuni tratti, corrispondenti al parapetto dell'antico cammino di ronda, oggi sono crollati. Inoltre, nel caso delle ultime due torri sul lato sudorientale, si sono anche riscontrate tracce di gradini in pietra che, innestandosi nella muratura, dovevano consentire l'accesso alla parte sommitale di queste. Delle scale che permettevano di raggiungere il cammino di ronda, invece, non è stata rinvenuta alcuna traccia.

Un fossato doveva, infine, correre tutt'attorno al recinto, e questo probabilmente si estendeva nello spazio compreso tra i due banchi di roccia presenti. L'ingresso, invece, viene ipotizzato in asse rispetto al percorso che storicamente collegava questo castello con quello di Lucugnano, a ovest, e quelli di Tricase e Caprarica, a est e a sud. 

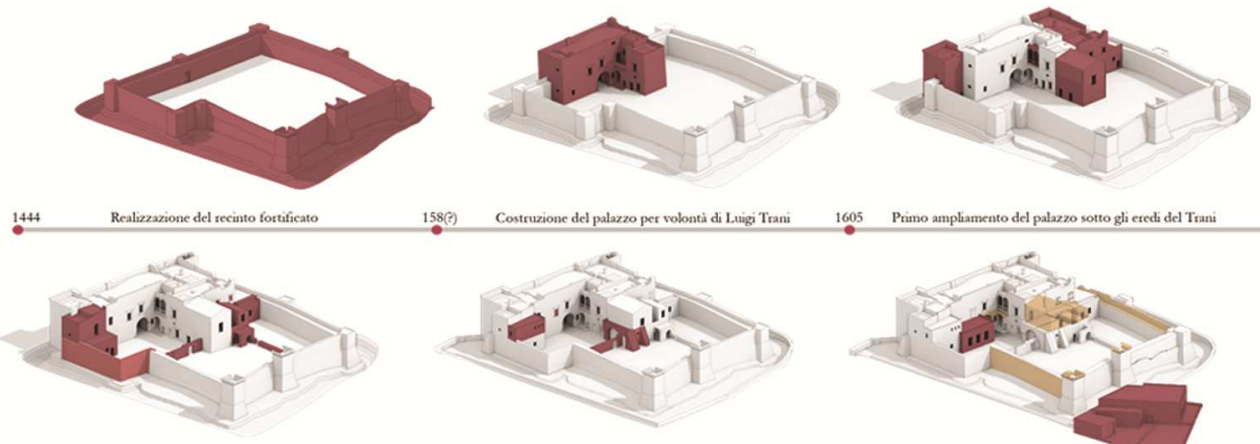

58(?) Costruzione del palazzo per volonti di Luigi Trani

605 Primo ampliamento del palazzo sotto gli eredi del Trani 1653
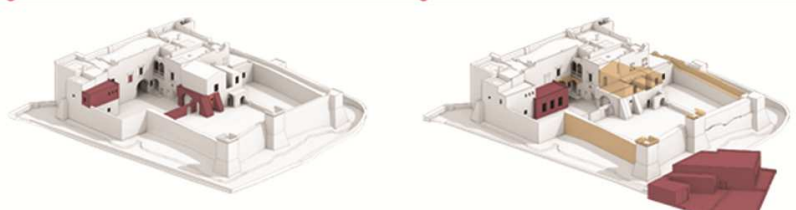

Fig. 1. Fasi storico-costruttive (Alessandra Ponzetta, 2015).

Le caratteristiche descritte inseriscono a pieno titolo questo castello nella tipologia a recinto, diffusasi nella parte meridionale della penisola salentina tra il XII e il XV secolo ${ }^{6}$; il clima di terrore e di insicurezza dovuto alle numerose scorrerie genera un'esigenza di difesa collettiva e la creazione di un luogo di rifugio nel quale convergere in caso di necessità. Come sostiene Raffaele Licinio (Licinio, 2010, p. 54), i castelli non sono tutti strutturati allo stesso modo, ma si adeguano alle funzioni e caratteristiche della comunità che li installa: si avranno così castelli con funzioni prettamente militari e altri progettati per la difesa di semplici coloni, chiese rurali o piccoli borghi. Per questo non troviamo castelli solo in località strategicamente importanti, ma anche in luoghi dove lo sfruttamento agricolo e la colonizzazione rurale sono gli aspetti predominanti. Questo può essere il caso di un piccolo centro come Tutino.

\section{La trasformazione in palazzo baronale}

Svariate e controverse sono le informazioni riportate dalle fonti relativamente al passaggio del feudo alla famiglia dei Trani, che trasformeranno il castello in residenza nobiliare. Molti studiosi parlano, infatti, di una donazione del feudo avvenuta nel XVI secolo da parte di Andrea Gonzaga a favore di Luigi Trani ${ }^{7}$. Maria Antonietta Visceglia (Visceglia, 2001, p. 233) scrive, invece, che "La famiglia che maggiormente "profittò" del disfacimento della signoria salentina dei Gonzaga fu la famiglia Trani, originaria di Terra di Bari e legata agli stessi Gonzaga - che acquistò con Luigi, Tutino nel 1594 e con Ottavio, Specchia di Preti, Tiggiano, Montesano, Melissano nel 1600 per 72.000 ducati". Le ricerche d'archivio condotte in questa sede hanno portato al ritrovamento del documento -ineditocon il quale Andrea Gonzaga, marchese di Specchia, ha il regio assenso alla vendita a favore del Dottor Luigi Trani del casale di Tutino nel $1582^{8}$. Tale scoperta porta a rivedere sia quanto sostenuto riguardo alla donazione, sia quanto detto in merito alla data di acquisizione del casale.

Inoltre, lo spostamento della data di acquisto in un periodo anteriore coincide con quanto si ricava dall'osservazione diretta del manufatto architettonico. La data di fondazione, infatti, è incisa sul muro di un vano del primo piano, parzialmente leggibile poiché nascosta in parte dietro lo stipite di una finestra, realizzata successivamente lungo il prospetto nord-occidentale. Qui si legge 158(?) con l'8 tagliato a metà, per cui si può stabilire con certezza solo il decennio di costruzione del palazzo. ${ }^{9}$

Risale, dunque, agli anni Ottanta del Cinquecento la realizzazione del palazzo baronale dei Trani a Tutino lungo il lato nord-occidentale del recinto fortificato. In questa prima fase di realizzazione del palazzo, esso doveva possedere una configurazione ad L, come suggerito dall' analisi stratigrafica condotta e dal confronto con esempi coevi (Fig. 1). L'iscrizione presente in facciata, che delimita assieme al cantonale d'angolo i confini del primo nucleo del palazzo e ne celebra la costruzione, appare in tutto simile a quella di 
palazzo Legari ad Alessano, realizzato nel 1536; occupa, infatti, la stessa posizione, utilizza la stessa formula celebrativa e gli stessi caratteri. Tale somiglianza, che si riscontra anche per gli apparati decorativi ed epigrafici delle aperture con altri palazzi di questo centro, conferma l'appartenenza storica del casale di Tutino alla Contea di Alessano, che si è manifestata anche nell'adozione di un linguaggio architettonicoartistico comune. A quell'epoca, inoltre, il castello di Tutino era dotato di uno stemma angolare, posizionato in corrispondenza del cantonale presente sul fronte nord-occidentale e raffigurante l'effige dei Trani, un drago che regge tra le zampe la testa di un bue, oggi abbandonato su una delle terrazze del castello. Un ulteriore stemma araldico della famiglia è incorniciato nel portale di gusto catalano-durazzesco sul prospetto principale, il cui tipo è ricorrente nei palazzi rinascimentali della provincia. Gli elementi menzionati appaiono, inoltre, nei coevi palazzi nobiliari di Lecce, che indubbiamente costituiscono un riferimento aulico per i centri minori di Terra d'Otranto e dove non è da escludere che abbiano operato anche le stesse maestranze.

\section{L'ampliamento sotto la famiglia Trani}

Nel tempo, rispetto al primo nucleo centrale del palazzo, sono state realizzate diverse aggiunte. L'incrocio delle informazioni documentarie e delle indagini dirette sull'edificio ha permesso di procedere alla loro individuazione (Fig. 1).

Alfredo Raeli (Raeli, 1981, p. 248) afferma che già nei primi anni del Seicento delle aggiunte vennero costruite a levante e ponente sotto gli eredi del Trani. Sulla base delle fattezze attuali si è riconosciuto questo ampliamento nei due volumi adiacenti al nucleo centrale lungo il fronte principale: il primo, a nord, si accosta in corrispondenza del cantonale d'angolo e prosegue sino ad inglobare il volume della cappella al piano primo; il secondo, a ovest, si addossa in prossimità della conclusione dell'epigrafe in facciata, impedendone così la lettura completa. L'unitarietà del fronte è tuttavia assicurata dall'inserimento di un basamento e di un coronamento a mensole continui lungo tutto lo svolgimento della nuova facciata. A questi ambienti si aggiunge un altro volume a sud-est, di cui oggi sopravvive solo il vano al piano terra. Del vano al piano primo possediamo riscontri materiali sull'edificio, oltre ad alcune testimonianze nelle fonti. Roberto Baglivo (Baglivo, 2001, p. 6) fa riferimento al lutto che colpì la famiglia nel 1605 , quando morirono, in pochi giorni, dapprima il figlio Andrea e poi la nuora Anna De Paulis del barone Orazio Trani. La lettura del testamento di quest'ultima ${ }^{10}$, redatto in punto di morte all'interno del palazzo, permette di identificare il vano in cui la malata giaceva. Quell'ambiente oggi non esiste più, essendo stato demolito; si tratta della seconda stanza, al piano superiore, che si raggiunge dal pianerottolo di arrivo della scala monumentale, varcando l'ingresso sul lato sud ${ }^{11}$. In questo stesso periodo dev'essere avvenuta la sistemazione della loggia come appare attualmente -secondo modelli riscontrati anche ad Alessano- con la realizzazione di due volte a crociera che intercettano le modanature della finestra dell'ammezzato, realizzata pertanto in precedenza.

\section{Le successive modifiche al castello/palazzo}

Il declino del castello di Tutino comincia a partire dal 1653, anno in cui Francesco Trani vendette a Stefano Gallone, principe di Tricase, il feudo di Tutino ${ }^{12}$. Alfredo Raeli (Raeli, 1981, pp. 248-249) racconta che "Sotto i principi Gallone il castello di Tutino fu trasformato in masseria, ed il paese andò sempre decadendo". Roberto Baglivo (Baglivo, 2001, p. 6) scrive inoltre che "il palazzo baronale, il giardino retrostante ed i magazzini di sotto, venivano concessi in affitto assieme agli atri beni feudali e burgensatici ricadenti nel feudo di Tutino. Solo un quarto, dei tre quarti, dello stesso palazzo rimaneva nella libera disponibilità del proprietario, spesso era quell'ala che comprende alcune stanze superiori dal lato della fontana pubblica dove esisteva una piccola cappella". Tale circostanza è confermata da un documento conservato nell'archivio dei principi Gallone: si tratta della sentenza di un giudice di pace contenuta in una raccolta di documenti datati tra il 1796 e il 1815 , dove è citato un tale Oronzo Panico, affittuario della masseria "Castello" in Tutino (Giorgi, 2001, p. 166). Lo stesso Cosimo De Giorgi (Giorgi, 1882-1888, p. 
166), quando il palazzo apparteneva a Luigia Gallone, ultima nobile proprietaria del castello, figlia del principe di Tricase e moglie del marchese Augusto Imperiali, dei principi di Francavilla Fontana, scrive che "Il cortile in parte è stato cangiato in orto, in parte a stalla da buoi. Un contadino abita il pian terreno, e il piano superiore è stato convertito in magazzino; le galline e i maiali vi sguazzano come in una reggia!" testimoniando l'incuria nel quale versava il complesso architettonico.

Si ipotizza che in questo periodo siano stati realizzati ulteriori ambienti funzionali all'utilizzo del castello-palazzo come masseria (Fig. 1). Sul fronte sud-occidentale, ad esempio, deve essere avvenuto un rimaneggiamento del muro perimetrale, che costituiva l'originaria cinta del castello, attraverso l'aggiunta di una colombaia. Una serie di modifiche, inoltre, hanno interessato le ultime due stanze al piano primo sul versante nord-orientale. In un primo momento, infatti, dev'essere stata realizzata all'interno del muro perimetrale sud-orientale una scala che permetteva di accedere alla quota delle terrazze e di raggiungere, attraverso un corridoio realizzato ispessendo la muratura sottostante, un vano costruito contestualmente al di sopra della cappella del piano primo. In un momento successivo, in occasione del rifacimento del solaio di copertura, quando sono stati inseriti i pilastri per la realizzazione delle nuove volte, la scala è stata murata e le due aperture sul fronte nord-est di questi vani sono state tamponate, collocandosi nel punto in cui dovevano essere inseriti tali sostegni.

Nel Novecento il castello fu acquistato dalla famiglia Caputo (Raeli, 1981, p. 249) ed adibito interamente ad opificio per la lavorazione del tabacco $^{13}$ sino agli anni Sessanta del XX secolo, quando fu abbandonato.

In questi anni il castello-palazzo subì altre trasformazioni legate alla variazione della sua destinazione d'uso (Fig. 1). Esse hanno riguardato tanto la realizzazione di nuovi volumi, facilmente identificabili per la copertura in laterocemento, quanto la risistemazione di quelli esistenti in vista della nuova funzione, come l'inserimento di servizi igienici e di impianti e apparecchiature per l'essiccazione delle foglie di tabacco, oppure l'asportazione di una porzione di volta a botte in un vano al piano terra per permettere un più rapido trasferimento dei materiali, in fase di lavorazione, al corrispondente ambiente al piano primo. All'esterno dell'edificio, invece, venne realizzato il muretto a secco di recinzione del fossato, che appare già in una cartolina del 1903 (Sabato, 1994, p. 243). Sempre in questa fase, probabilmente in previsione della demolizione di alcuni vani al piano primo, vennero realizzati dei contrafforti lungo la parete sudorientale dei corrispondenti ambienti al piano terra. Sino al 1930, infatti, sono documentati alcuni vani al piano primo, raffigurati nel disegno di Primaldo Coco (Coco, 1930, p. 66 ), che non compaiono più nelle planimetrie catastali del $1940^{14}$. È in questo lasso temporale che deve esserne avvenuta la demolizione. Inoltre, in queste stesse planimetrie non risulta ancora il vano al piano terra coperto da un solaio in latero-cemento, che viene accatastato in un momento successivo, come variante all'impianto originario $^{15}$. Tra questo ambiente e l'ala nordorientale del palazzo venne poi realizzata una tettoia, di cui restano oggi le sole tracce sulle pareti dei vani adiacenti, essendo stata rimossa in data imprecisata. Esternamente, invece, un'abitazione fu costruita a ridosso del fossato del castello (il cui accatastamento risale al 1971), intaccandone così la lettura unitaria.

Nonostante il complesso palaziale non sia frutto di un unico intervento edilizio e quindi sia caratterizzato da una stratificazione di elementi successivi, si può constatare l'impiego di tecniche costruttive analoghe; le sostanziali, se pur lievi, differenze si riscontrano nelle dimensioni dei conci impiegati, disposti in filari orizzontali di altezza costante. Tutte le murature evidenziano l'uso esclusivo di materiale lapideo, in particolare di pietra calcarea, in continuità con l'area geografica di riferimento.

\section{Conclusioni}

L'analisi sin qui condotta, attraverso la ricerca documentaria e sul campo, fornisce nuovi utili elementi per la conoscenza del patrimonio pugliese. La ricostruzione delle fasi storicocostruttive dell'edificio, per cui ci si è avvalsi 
anche di un'attenta lettura stratigrafica, in alcuni casi ha fornito conferme rispetto alla bibliografia di riferimento, in altri casi ha permesso di confutare tesi che non trovavano riscontro nella consistenza materica stessa del monumento. Le informazioni emerse hanno tratteggiato un rapporto inscindibile tra il castello di Tutino ed il suo territorio di appartenenza, che ne ha influenzato tanto l'origine, quanto condizionato l'evoluzione nel tempo. Esso è espressione della cultura del luogo, di cui racconta la storia attraverso il linguaggio architettonico e artistico. Tale lettura permette inoltre l'istituzione di confronti tipologici, morfologici e costruttivi con altri casi studio, contribuendo a fornire un quadro di riferimento sempre più ampio e dettagliato, in particolare nella prospettiva ultima del restauro.

\section{Note}

${ }^{1}$ Quanto qui esposto è l'esito del lavoro di tesi magistrale in restauro architettonico dal titolo "Il castello di Tutino (Le). Dall'analisi al progetto di restauro", svolto assieme ai colleghi Alberga, F.; Capurso, P.; Ciavarella, M.; Grato, A.; Losito, A.F.; Muscogiuri, A., presso il Politecnico di Bari nell'a.a. 2013/14 e coordinato dalla prof.ssa arch. Rossella de Cadilhac.

${ }^{2}$ Il casale di Caprarica del Capo (XII-XIII secolo) seguì le vicende della contea di Alessano, a cui lo stesso Tutino era legato (Montefusco, 1994, p. 35). Anche in questo caso, come testimonia un documento datato 1512 , è sotto i del Balzo che si registra la presenza di un castello (Accogli, 2006, p. 44), ristrutturato nel 1524 ad opera dell'architetto Antonio Renna all'indomani del sacco di Otranto (Cazzato, 1997, p. 36).

${ }^{3}$ Si tratta della richiesta del feudatario Raimondo del Balzo al re Alfonso I di essere investito, alla morte del padre Giacomo, dei beni posseduti da questo durante la sua vita (Ferraro, 2001).

${ }^{4}$ Raimondello del Balzo Orsini (1361-1406), figlio di Roberto Orsini e Sveva del Balzo, fu principe di Taranto dal 1393. Questo aveva sposato nel 1384 Maria d'Enghien, contessa di Lecce, che alla sua morte divenne regina consorte di Napoli di Ladislao I d'Angiò Durazzo. A lui seguirà Giovanni Antonio del Balzo Orsini (1386-
1463), ultimo principe di Taranto, cui si attribuiscono importanti opere militari (Bari, Lecce, etc), nelle quali aveva trasferito la sua esperienza di guerra (Visceglia, 1988, pp. 167-181).

${ }^{5}$ Dalle ricerche bibliografiche si apprende che "Nel 1480, dopo la presa di Otranto, i Turchi saccheggiarono Salete (oggi Depressa) e molti fuggiaschi di detto casale si ripararono a Tutino, che in tal modo si ingrandi”" (Raeli, 1981, p. 247; Ruotolo, 1952, p. 298).

${ }^{6}$ Ricercando le matrici tipologiche dei castelli salentini, emerge come schema originario ricorrente quello del recinto: un circuito murario con torrioni perimetrali ed un unico vano di accesso. I castelli di Tutino, Caprarica del Capo, Fulcignano, Patù, Melpignano, rientrano in questa tipologia e sono quelli che tuttora consentono una lettura più immediata di tale impianto. (Cazzato, Cazzato, 2005, p. 87).

7 Giacomo Arditi parla di una donazione del feudo da parte di Andrea Gonzaga a favore di Luigi Trani nel XVI secolo (Arditi, 1979, p. 631). Alfredo Raeli precisa che Andrea Gonzaga vendette tutti i feudi della contea (di Alessano) ad Ettore Brayda, marchese di Lavello, ad eccezione di Tutino che donò a don Luigi Trani (Raeli, 1981, p. 247).

8 Si tratta del documento conservato presso l'Archivio General de Simancas, Titulos y privilegios de Napoles (sec. XVI XVIII), p. 270.

9 Tale data era già visibile nel 1888 , quando Cosimo De Giorgi scrive "Infatti in una stanza del piano superiore si legge l'epoca di fondazione (158...) dietro lo stipite di una finestra che mette sulla facciata" (Giorgi, 1882-1888, p. 165).

${ }^{10}$ Il documento è conservato presso l'Archivio di Stato di Lecce, sezione notarile, 109\#1, 1605, fol. $51 \mathrm{r}$.

${ }^{11}$ Si legge, infatti, “[...] ipsam Annam in superiori parte ipsorum domorum in secunda camera, ad quam super ultimam, et supremum seule gradum, ingressus $\dagger$ ad sinistram, in lecto iacentem [...]" (ASL, sezione notarile, 109\#1, 1605, fol. 51 r.).

${ }^{12}$ L'atto è stipulato dal notaio G. A. Zaccaria di Poggiardo (e rogato a Diso) il 14 agosto 1653 e 
ratificato dal notaio M.G. Pitigliano di Napoli il 26 dello stesso mese (Raeli, 1981, p. 248; Giorgi, 2001, p. 35).

${ }^{13}$ Rossella Barletta riporta tra le ditte concessionarie per la lavorazione del tabacco nel 1929, a Tricase, i nomi di Caputo Domenico e Alessandro (Barletta, 1994, p. 127).
${ }^{14}$ Agenzia del Territorio, Ufficio provinciale di Lecce, scheda 03681046, planimetria catastale

15 Agenzia del Territorio, Ufficio provinciale di Lecce, scheda 03681038, planimetria catastale.

\section{Bibliography}

Accogli, F. (2006). I cinque castelli della Terra di Tricase, Edizioni dell' Iride, Tricase.

Arditi, G. (1979). La corografia fisica e storica della provincia della terra d'Otranto, Arnaldo Forni Editore, Bari.

Baglivo, R. (2001). "Ricordi di un castello", Nuove Opinioni. Mensile indipendente di vita e cultura, XXIV, 2, p. 6.

Barletta, R. (1994). Tabacco tabaccari e tabacchine nel Salento. Vicende storiche, economiche e sociali, Schena Editore, Fasano.

Cazzato, M. (1997). Guida ai Castelli Pugliesi, la provincia di Lecce, Congedo Editore, Galatina.

Cazzato, M.; Cazzato, V. (2005). "Strumenti per la lettura dei centri urbani del Salento", in Cazzato, V.; Guaitoli, M., eds., Insediamenti del Salento dall'antichità all'età moderna, Congedo Editore, Galatina, pp. 79-88.

Coco, P. (1930). Porti castelli e torri salentine, Istituto di Architettura Militare, Roma.

Ferraro, A. (2001). Salignano e i suoi documenti, Edizioni dell'Iride, Tricase.

Fuzio, G. (1979). "Castelli: tipologie e strutture”, in Fonseca, C.D., ed., La Puglia tra Medioevo ed età Moderna. Città e Campagna. Civiltà e culture in Puglia, Electa, Milano, vol. III, pp. 118-201.

Giorgi, C. de. (1882-1888). La provincia di Lecce. Bozzetti di viaggi, Editore Giuseppe Spacciante, Lecce, vol. I.

Giorgi, D.L. de. (2001). L'archivio dei principi Gallone. Documenti dello "Stato" di Tricase, Edizioni dell'Iride, Tricase.

Licinio, R. (2010). Castelli medievali. Puglia e Basilicata: dai Normanni a Federico II e Carlo I d' Angiò, Caratteri Mobili Editori, Bari.

Montefusco, L.A. (1994). Le successioni feudali in Terra d'Otranto, la provincia di Lecce, Istituto Araldico Salentino, Lecce.

Raeli, A. (1981). Aneddoti di storia tricasina, Congedo Editore, Galatina.

Ruotolo Mons, G. (1952). Ugento - Leuca - Alessano. Cenni storici e attualità, Edizioni Cantagalli, Siena.

Sabato, A. (1994). La Puglia dei Castelli, Edizioni del Grifo.

Visceglia, M.A. (1988). Territorio feudo e potere locale. Terra d'Otranto tra Medioevo ed età Moderna, Guida editori, Napoli. 